\title{
El reto de COVID-19 respecto a la responsabilidad social de las escuelas de medicina: nuevas perspectivas profesionales y
} humanas

\author{
Luis F. Abreu-Hernández, ${ }^{1}$ Jorge E. Valdez-García, ${ }^{2}$ Ramón I. Esperón-Hernández ${ }^{3}$ y \\ Silvia L. Olivares-Olivares ${ }^{2 *}$ \\ ${ }^{1}$ Universidad Nacional Autónoma de México, Facultad de Medicina, Cuidad de México; ${ }^{2}$ Escuela de Medicina y Ciencias de la Salud, Tecnológico \\ de Monterrey, Nuevo León; ${ }^{3}$ Universidad Westhill, Facultad de Medicina, Cuidad de México. México
}

\section{Resumen}

Las escuelas de medicina desempeñan un papel central en la acumulación y desarrollo del conocimiento profesional, por lo cual poseen privilegios y recursos que se justifican solo en la medida en que los retribuyan a la comunidad, en particular a los más necesitados. La responsabilidad social de las escuelas de medicina se centra en los servicios formativos, asistenciales y de investigación que ofrecen. Los principios de la educación médica y la estructura propuesta por el Informe Flexner están en crisis debido a la pandemia de COVID-19 y se requiere la redefinición del contrato social. El presente documento ofrece una propuesta de responsabilidad social de las escuelas de medicina que incluye previsión de las necesidades de la comunidad, atención interprofesional centrada en el paciente, formación de profesionales en el área de salud y colaboración entre instituciones. Resalta la necesidad de una institución consciente que encuentre nuevos espacios de entrenamiento diferentes al hospitalario, donde se atienda a cada paciente de forma personalizada, con modelos formativos interprofesionales que consideren al alumno como persona que cuida de sí misma en colaboración abierta con las organizaciones. Los líderes deben actuar ya porque es su responsabilidad social y porque es lo correcto.

PALABRAS CLAVE: Responsabilidad social. Atención centrada en el paciente. Autocuidado. Equipo interprofesional. Currículo integrado.

\section{COVID-19 challenge with regard to medical schools social responsibility: new professional and human perspectives}

\begin{abstract}
Medical schools play a central role in the compilation and development of professional knowledge, which is why they have privileges and resources that are justified only to the extent that they use them to serve the community, particularly those who are most in need. Medical schools social accountability focuses on the training, healthcare provision and research services they offer. The principles of medical education and the structure proposed by the Flexner Report are in crisis due to the COVID-19 pandemic, and redefinition of the social contract is required. This document offers a proposal for medical schools social accountability that includes anticipation of the needs of the community, patient-centered inter-professional care, training of people in the area of health and collaboration between institutions. It highlights the need for a conscious institution that finds new training spaces other than hospitals, where each patient is cared for in a personalized way, with inter-professional training
\end{abstract}

Correspondencia:

*Silvia L. Olivares-Olivares

E-mail: solivares@ @ec.mx
Fecha de recepción: 20-05-2020

Fecha de aceptación: 29-05-2020

DOI: 10.24875/GMM.20000306
Gac Med Mex. 2020;156:311-316

Disponible en PubMed

www.gacetamedicademexico.com

0016-3813/๑ 2020 Academia Nacional de Medicina de México, A.C. Publicado por Permanyer. Este es un artículo open access bajo la licencia CC BY-NC-ND (http://creativecommons.org/licenses/by-nc-nd/4.0/). 
models that consider the student as a person who takes care of him/herself in open collaboration with organizations. Leaders must act now because it is their social accountability and because it is the right thing to do.

KEY WORDS: Social accountability. Patient-centered care. Self-care. Inter-professional team. Integrated curriculum.

\section{Introducción}

Desde sus estudios iniciales, Freidson ${ }^{1}$ señalaba que la medicina, como profesión, se sustenta en una autonomía garantizada y reconocida por el Estado, lo cual conduce a la creación de una especie de monopolio, producto del reconocimiento de los beneficios que aporta esta disciplina. Este contrato social se concede a cambio de un profesionalismo y un comportamiento ético que incluye la empatía, la compasión, la honestidad, la integridad, el altruismo y la excelencia profesional.

En las últimas décadas, la autonomía profesional ha sido constantemente limitada porque se involucran organismos burocráticos altamente jerarquizados que pretenden controlar los costos o incrementar las ganancias. ${ }^{1}$ La práctica de la medicina se ha sometido a todo tipo de lineamientos, guías, protocolos y estándares adoptados de los procesos de producción, en detrimento de la relación con el paciente. ${ }^{2,3}$ Esta situación ha derivado en un alejamiento de las expectativas de los individuos, las familias y las comunidades, que lejos de promover el sentido de servicio, soslaya las necesidades sociales.

A pesar de lo anterior, el contrato social de las escuelas de medicina indica que estas instituciones tienen la responsabilidad de influir en los cambios en el sistema de atención de la salud, para que evolucione a un modelo eficaz, eficiente, accesible, equitativo y sostenible. Para lograrlo se formularon principios de la educación médica congruentes con las declaraciones de la Organización Mundial de la Salud ${ }^{4}$ y el Consenso General de Responsabilidad Social de las Escuelas de Medicina. ${ }^{5} \mathrm{La}$ pandemia de COVID-19 constituye un reto directo a estas premisas que, si bien han evolucionado a lo largo de los años, hoy son obsoletas e insuficientes. ${ }^{6}$

\section{La responsabilidad social de las escuelas de medicina en la actualidad}

La Organización Mundial de la Salud define la responsabilidad social de las escuelas de medicina como la obligación de dirigir sus actividades de educación, investigación y servicios a la solución de los problemas prioritarios de salud en el lugar donde les corresponde servir. ${ }^{4}$ Esta necesidad se hizo evidente al verificar que diversas escuelas de medicina obtenían bajas puntuaciones en su vinculación social. ${ }^{7}$

En esa misma línea de pensamiento, el Consenso Global sobre Responsabilidad Social de las Escuelas de Medicina (GCSA, Global Consensus for Social Accountability of Medical Schools) señala que las escuelas de medicina deben dar respuesta a las necesidades de salud actuales y futuras, reorientar sus prioridades educativas y de investigación, reforzar su asociación con los grupos de interés y utilizar la acreditación basada en el desempeño. ${ }^{4}$ Parte de estas responsabilidades se cumplen con la formación de los profesionales de la salud a partir de una estructura derivada del Informe Flexner, la cual ha sido aceptada durante décadas. ${ }^{8}$

\section{Principios básicos de la educación médica}

El modelo dominante en la educación médica se organiza en torno a cuatro principios básicos, los cuales son insuficientes en las condiciones actuales de la pandemia de COVID-19 (Tabla 1).

\section{Primer principio: concentración de la instrucción en los niveles superiores del sistema de salud}

Desde el Informe Flexner se ha perpetuado una estructura curricular que establece la enseñanza de la ciencia seguida del aprendizaje clínico; una primera etapa de aprendizaje sobre contenido biomédico y después la enseñanza en los hospitales junto a la cama del paciente. $^{9}$ Lo anterior contradice lo que establece el GCSA: "la facultad de medicina reconoce que un sistema de salud sólido debe estar basado en un enfoque firme de atención primaria de la salud". ${ }^{5}$ Estudios recientes demuestran que la medicina en casa tiene mejores resultados clínicos, especialmente en pacientes con comorbilidades; ${ }^{10}$ sin embargo, los alumnos excepcionalmente dedican tiempo a intervenciones de atención domiciliaria o en escuelas y centros de trabajo.

Reto 1. La aparición de COVID-19 ha requerido la atención intradomiciliaria a través de telemedicina, 
Tabla 1. Principios de la educación médica en los que se enfrentan retos evidenciados por la pandemia de COVID-19

\begin{tabular}{l|l}
\hline $\begin{array}{l}\text { Principios de la educación médica } \\
\begin{array}{l}\text { Concentración de la instrucción en los } \\
\text { niveles superiores del sistema de salud }\end{array}\end{array}$ & $\begin{array}{l}\text { La emergencia requiere prevención y manejo de los enfermos en el hogar. El hospital deja de } \\
\text { ser el ambiente propicio para el tratamiento de pacientes y la enseñanza. }\end{array}$ \\
$\begin{array}{l}\text { Segmentación y desagregación de } \\
\text { componentes }\end{array}$ & $\begin{array}{l}\text { El virus afecta a múltiples órganos y niveles de organización. Las comorbilidades e } \\
\text { indefiniciones demandan una acción coordinada de varios servicios clínicos, incluyendo su } \\
\text { polifuncionalidad. }\end{array}$ \\
\hline $\begin{array}{l}\text { Homogeneidad de los pacientes } \\
\text { Generación centralizada del } \\
\text { conocimiento }\end{array}$ & $\begin{array}{l}\text { No hay patrones únicos en la evolución de la enfermedad. } \\
\text { La agenda de investigación incluye áreas que no han sido de mayor interés para los }\end{array}$ \\
\hline
\end{tabular}

\begin{abstract}
paramedicina comunitaria y servicios de atención virtuales. ${ }^{11}$ El correcto manejo implica la orientación médica a distancia, realizar pruebas para la detección temprana de síntomas e, incluso, utilizar el monitoreo capilar de oxigenación para evitar complicaciones y la hospitalización de los pacientes. ${ }^{12,13} \mathrm{~A}$ lo anterior se debe añadir la prevención, la educación de las comunidades, el aislamiento de casos y el seguimiento de los contactos como eje del sistema de salud, en los cuales el hospital solo debe ser un mecanismo de apoyo.
\end{abstract}

\section{Segundo principio: segmentación y desagregación de componentes}

La propuesta de Flexner postula una medicina científica que guía la práctica clínica unidireccional y linealmente. ${ }^{14}$ Los futuros médicos son formados en la orientación reduccionista, que descompone a las personas en partes para localizar las fallas y corregirlas. Esta tradición disciplinar genera dificultades para la integración curricular. ${ }^{15}$ Los estudiantes de medicina aprenden contenidos y prácticas independientes, los cuales experimentan aislada y descoordinadamente en servicios clínicos, bajo el concepto de privilegiar la causalidad inmediata.

Reto 2. El paradigma lineal está en crisis, puesto que el conocimiento se genera tanto en el laboratorio como en la práctica clínica, conforme el concepto de la medicina traslacional. ${ }^{16}$ COVID-19 actúa en todos los niveles: molecular, celular, órganos, aparatos y sistemas, lo cual requiere el manejo por equipos interdisciplinarios y polifuncionales. ${ }^{17}$ La emergencia requiere articular expertos de los diferentes niveles de atención para empoderar la acción en las comunidades. ${ }^{18}$

\section{Tercer principio: homogeneidad de los pacientes}

Los alumnos son educados en el paradigma de que todos los pacientes responden de la misma manera a las mismas enfermedades y se tratan de la misma forma. Este enfoque ha sido denominado por Montori ${ }^{3}$ como "medicina industrializada", porque actúa con parámetros característicos de la producción fabril. Esta orientación implica ignorar el contexto y evitar la adaptación al caso concreto. Berwick ${ }^{19}$ ha demostrado el daño que puede resultar al eliminar dichas variaciones.

Reto 3. Una persona con COVID-19 puede cursar sin manifestaciones o desarrollar complejos fenómenos moleculares, de inflamación, tormenta de citocinas y coagulación intravascular, hasta desembocar en falla orgánica múltiple. ${ }^{12} \mathrm{La}$ incertidumbre y la diversidad brindan mayores oportunidades de aprendizaje, como el basado en retos. ${ }^{20,21}$

\section{Cuarto principio: generación centralizada del conocimiento}

Los centros académicos capaces de hacer investigación están centralizados y articulados con el complejo biomédico-industrial líder que dirige la investigación médica en el mundo. Los estudiantes solo son instruidos en localizar, leer y valorar la calidad de un artículo de investigación. ${ }^{22}$ Esta realidad carece de formación metodológica y de oportunidades para realizar investigación o hacer innovaciones para mejorar la calidad de la atención. ${ }^{23}$

Reto 4. Cada miembro de la comunidad académica está aprendiendo sobre el SARS-CoV-2 en múltiples fuentes, en las cuales puede investigar desde 
la biología molecular del virus, la fisiopatología, la utilidad de fármacos, el manejo clínico o las medidas preventivas. ${ }^{24}$ Actualmente, las investigaciones pueden desarrollarse en los tres niveles de la atención y de forma descentralizada en multitud de países y grupos internacionales, gracias a los avances de la informática. Están adquiriendo relevancia otras modalidades de publicación rápida como los preprints, a los cuales la comunidad científica tiene acceso de forma inmediata y gratuita. ${ }^{25}$

\section{El perfil tradicional de los egresados}

Desde el año 2000, varios países y organismos internacionales de educación médica se han esforzado por establecer las competencias del médico moderno. ${ }^{26,27}$ Sin embargo, este trabajo de diseño de competencias se llevó a cabo bajo un supuesto diferente al mundo que hoy se colapsa por la pandemia. El cambio es tan radical que algunos apuntan a dos épocas distintas, antes y después del COVID-19, A. C. y D. C. por sus siglas. ${ }^{28,29}$

Ante este nuevo escenario se debe cuestionar si las competencias médicas son suficientes para la denominada nueva normalidad. Para este análisis se utilizan las competencias del médico general mexicano establecidas por la Asociación de Facultades y Escuelas de Medicina (AMFEM): ${ }^{30}$

1. Dominio de la atención médica general.

2. Dominio de las bases científicas de la medicina.

3. Capacidad metodológica e instrumental en ciencias y humanidades.

4. Dominio ético y del profesionalismo.

5. Dominio de la calidad de la atención médica.

6. Dominio de la atención comunitaria.

7. Capacidad de participación en el sistema de salud.

Esta conceptualización coincide con las competencias establecidas en países europeos y por las asociaciones médicas, por lo que representa el pensamiento y significado de la medicina de quienes fueron formados en décadas pasadas. ${ }^{26} \mathrm{Al}$ revisar estas competencias destaca que se privilegia la salud, seguridad y expectativas del paciente, ignorando al médico en formación como persona. Al contrastarlas con los principios del GCSA se identifica que hay consistencia respecto al enfoque individuo-familia-comunidad, la promoción de la salud, la educación para la salud, la prevención y protección específica de las enfermedades, el enfoque epidemiológico y la atención de los derechos humanos, entre otros. ${ }^{30}$

Sin embargo, ante la pandemia de COVID-19 se hace evidente la necesidad de una nueva competencia que considere al estudiante como una persona

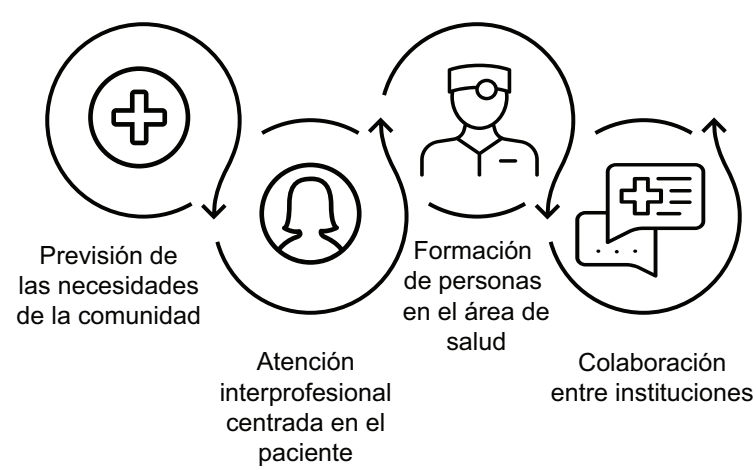

Figura 1. La nueva responsabilidad social de las escuelas de medicina.

con necesidades y expectativas. ${ }^{* 31,32}$ Esta perspectiva se incluye parcialmente en la competencia denominada "dominio ético y del profesionalismo", en la cual se identifica el apartado "compromiso consigo mismo", que se centra en la reflexión y el análisis personal. ${ }^{30}$

Esta visión sesgada de beneficio exclusivo a la sociedad se ha interpretado como heroísmo, servilismo y caridad, lo cual ha incidido en contagios, suicidios, limitaciones en la libertad de conciencia y hasta sarcasmo, por una remuneración digna por los servicios otorgados. ${ }^{33,34}$ Los mismos alumnos carecen de una visión centrada en el paciente debido a la constante amenaza a su identidad profesional. ${ }^{35}$ La pandemia de COVID-19 ha destacado la necesidad de formación en los siguientes aspectos: fortaleza emocional, autoprotección contra riesgos inherentes a la profesión y autocuidado de la salud personal y la propia economía. ${ }^{34}$

\section{La nueva función social de las escuelas de medicina a partir de la pandemia}

La responsabilidad social se reorienta y reconceptualiza ante la contingencia de COVID-19. Se propone un modelo que refleje las funciones de la previsión de las necesidades de la comunidad, la atención interprofesional centrada en el paciente, la formación de personas en el área de salud y la colaboración entre instituciones (Figura 1).

Clithero-Eridon, Albright y Ross ${ }^{36}$ encuentran que - desde la perspectiva de los estudiantes de medicina, mentores y médicos-, la responsabilidad social significa primordialmente dar servicio a la comunidad,

* Olivares S, Rivera N, López M, Turrubiates M. Etapas de la identidad para ser profesionista: Evolución de las expectativas de los retos académicos a lo largo de la carrera. Formación Universitaria, 2020. En prensa. 


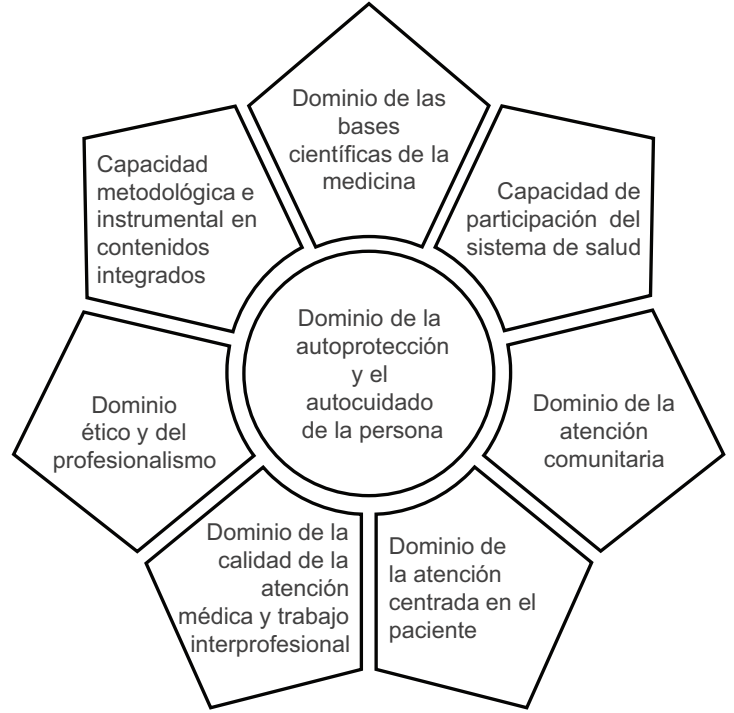

Figura 2. Modelo de competencias para el médico general mexicano adaptado del modelo de la Asociación de Facultades y Escuelas de Medicina. $^{30}$

asegurando la salud y el bienestar. Por años se ha identificado el hospital como el escenario más importante de aprendizaje para las escuelas de medicina, sin embargo, según Woolliscrof, ${ }^{37}$ tal vez sea momento de considerar el "hospital en casa" y la comunicación virtual como recursos efectivos y eficientes para la atención clínica y la educación. La pandemia está demandando aprender continuamente, actuar en forma creativa frente a lo incierto y desconocido, así como evaluar críticamente la propia actuación. ${ }^{38}$

Los nuevos modelos requieren ser estructurados desde la realidad centrada en el paciente y la comunidad, con un enfoque integral y personalizado. ${ }^{39} \mathrm{La}$ estratificación de las disciplinas y las profesiones se desdibuja junto con la línea que divide las fronteras del conocimiento entre las mismas. La educación interprofesional se convierte en una necesidad fundamental para colaborar en ambientes de incertidumbre. ${ }^{40}$ Este tipo de enseñanza y colaboración permite a los integrantes del equipo tomar decisiones como iguales para asegurar la calidad y seguridad de la atención clínica. ${ }^{41}$ La intervención interprofesional se convierte en una oportunidad para encontrar nuevas estrategias para el tratamiento de los pacientes con COVID-19. ${ }^{18}$

Respecto a la función social de formar profesionales de salud es indispensable agregar una competencia: el dominio de la autoprotección y el autocuidado de la persona, donde la persona puede ser el paciente, el alumno, el docente o el médico (Figura 2).
El compromiso de la institución debe ampliarse hacia programas educativos que garanticen la seguridad, la integridad física, la salud, la libertad, el desarrollo profesional, el prestigio, la dignidad, el cuidado de las emociones, la autonomía y las relaciones. ${ }^{26}$ Según Kofman, ${ }^{42}$ la empresa consciente es aquella con consciencia tanto del mundo interior como del exterior. Esto implica atender a la sociedad sin descuidar a la comunidad académica. Los programas educativos actuales para los profesionales de la salud están siendo insuficientes para abordar una realidad multifactorial en el nuevo paradigma complejo..$^{43}$ Lo único constante de los últimos años es la incertidumbre, el azar, la indeterminación y la emergencia, lo cual se hace tangible con la pandemia de COVID-19.

Los profesionales del presente y del futuro deben ser formados para enfrentar las adversidades de forma creativa e innovadora. La emergencia sanitaria ha impulsado a utilizar innovaciones antes poco exploradas como la atención clínica a distancia, la determinación de diagnósticos e intervenciones terapéuticas con técnicas avanzadas y la educación virtual, lo cual está transformando la manera de conceptualizar la educación médica. ${ }^{37,44,45}$

COVID-19 ha demostrado la incapacidad para hacer converger los programas educativos y los sistemas de salud. Hay una necesidad imperativa de atender a las comunidades desde las escuelas de medicina a través de modelos abiertos de colaboración entre instituciones, que incluyan a la educación superior, el sector salud, la iniciativa privada y el gobierno. ${ }^{5}$ Según Boelen, Dharamsi y Gibbs, ${ }^{46}$ la escuela de medicina socialmente responsable trabaja en dicha asociación para lograr impactar en la salud de las personas y lo demuestra con resultados relevantes, de alta calidad. Torre ${ }^{47}$ advierte acerca de la necesidad de convergencia de los sectores público y privado para atender las necesidades biológicas de salud y, además, las económicas, que se incrementan a medida que avanza la pandemia.

\section{Conclusión}

A medida que avanza una nueva era hacia la atención centrada en el paciente, se evidencia la necesidad de que las escuelas de medicina del país articulen y expliquen las percepciones del público y sus obligaciones internas y externas. Las escuelas de medicina deben dejar de autoconceptualizarse bajo esquemas lineales y aislados para pasar a estructuras flexibles, integradas y activas, hacia una transformación que tenga un impacto directo en la salud de todos. 
Los líderes de las instituciones deben involucrar a la comunidad académica y otros grupos de interés de la sociedad en la planeación y ejecución acelerada de políticas, programas e intervenciones que inicien un nuevo sistema de salud y la educación médica D.C. Los líderes deben hacerlo ya, simplemente porque es lo correcto.

\section{Conflicto de intereses}

Los autores declaran no tener conflicto de intereses.

\section{Financiamiento}

Los autores no recibieron patrocinio para llevar a cabo este artículo.

\section{Responsabilidades éticas}

Protección de personas y animales. Los autores declaran que para esta investigación no se realizaron experimentos en seres humanos ni en animales.

Confidencialidad de los datos. Los autores declaran que en este artículo no aparecen datos de pacientes.

Derecho a la privacidad y consentimiento informado. Los autores declaran que en este artículo no aparecen datos de pacientes.

\section{Bibliografía}

1. Freidson E. Profession of medicine: a study of the sociology of applied knowledge. EE. UU.: Dodd, Mead \& Co.; 1970.

2. Topol E. Deep medicine: how artificial intelligence can make healthcare human again. EE. UU.: Basic Books; 2019.

3. Montori V. Why we revolt: a patient revolution for careful and kind care. The patient Revolution. EE. UU.: Rochester; 2017.

4. Boelen C, Heck J. Defining and measuring the social accountability of medical schools: WHO/HRH/95.5. Suiza: Division of Development of Human Resources for Health; 1995.

5. Boelen $\mathrm{C}$, Woollard R. Consenso global sobre la responsabilidad social. Edu Med. 2011;14:7-14.

6. Valdez-García JE, López-Cabrera MV, Jiménez MA, Díaz-Elizondo JA Dávila-Rivas JA, Olivares-Olivares SL. Me preparo para ayudar: respuesta de escuelas de medicina y ciencias de la salud ante COVID-19. Inv Edu Med. 2020;9:1-11.

7. Mullan F, Chen C, Petterson S, Kolsky G, Spagnola M. The social mission of medical education: Ranking the schools. Ann Inter Med. 2010;152:804-811.

8. Oriol Bosch A. De Flexner a Bolonia. Edu Med. 2010;13:193-195.

9. Dornan T. Osler, Flexner, apprenticeship and 'the new medical education'. J Roy Soc Med. 2005;98:91-95

10. Low LL, Vasanwala FF, Ng B, Chen C, Kheng HL, Yun ST. Effectiveness of a transitional home care program in reducing acute hospital utilization: A quasi-experimental study. BMC Health Serv Res. 2015;15:100.

11. Hollander JE, Carr BG. Virtually perfect? Telemedicine for Covid-19. N Engl J Med. 2020;382:1679-1681.

12. Pedersen SF, Ho YC. SARS-CoV-2: A storm is raging. J Clin Invest. 2020;130:2202-2205.

13. Chen GE, Wu D, Guo W, Cao Y, Huang D, Wang H, et al. Clinical and immunological features of severe and moderate coronavirus disease. $J$ Clin Invest. 2020;130:2620-2629.

14. Godin B. the linear model of innovation: The historical construction of an analytical framework. Sci Tech Hum Val. 2006;31:639-667.

15. Haramati A. Educating the educators: A key to curricular integration. Acad Med. 2015;90:133-135.
16. Valdés IP, Ramírez-Santana M, Basagoitía A, Testar X, y Vásquez J A Medicina traslacional e innovación en salud: mecanismos y perspectivas. Rev Med Chile. 2018;146:890-898.

17. Gutiérrez D. Prueba TecSalud tratamiento integral. El Norte (México). 2020 Abr 19.

18. Brazeau G. Leadership challenges and collaborative opportunities unveiled through COVID-19. Mashall J Med. 2020;6. DOI: 10.33470/23799536.1282

19. Berwick D. Eating soup with a fork. En: 19th Annual IHI National Forum. EE. UU; 2007.

20. Olivares-Olivares SL, López-Cabrera MV, Valdez-García JE. Aprendizaje basado en retos: una experiencia de innovación para enfrentar problemas de salud pública. Edu Med. 2018;19:230-237.

21. Kalinga EA, Ibwe KS, Mvungi $\mathrm{NH}$, Tenhunen $\mathrm{H}$. Active learning through smart grid model site in challenge based learning course. Syst Cybern Inform. 2018;16:53-64.

22. Rader $\mathrm{H}$. Bibliographic instruction or information literacy. Coll Res Libr News. 2019;51:18-20.

23. Collins $F$, Varmus $H$. A new initiative on precision medicine. $N$ Engl $J$ Med. 2105:372:793-795

24. Zhou D, Dai SM, Tong Q. COVID-19: A recommendation to examine the effect of hydroxychloroquine in preventing infection and progression. J Antimicrob Chemother. 2020.

25. Sánchez-Mendiola M. Educación médica y la pandemia: ¿aislarse o colaborar? Rev Inv Edu Med. 2020;9:1-3.

26. Esperón-Hernández R. Desarrollo de competencias para la toma de decisiones médicas basadas en la evidencia en estudiantes de medicina de pregrado. España: Universidad de Granada; 2014.

27. Frank J, Snell LS. CanMEDS 2015 physician competency framework. Canadá: Royal College of Physicians and Surgeons of Canada; 2015.

28. Friedman T. Our new historical divide: B.C. and A.C. the world before corona and the world after. The New York Times (EE. UU). 2020 Mar 17.

29. Moe M, Rajendran V. Dawn of the age of digital learning:an acceleration of trends that have been building for years. 2020 May 6. En: GSV Ventures [Internet]. EE. UU: GSV Ventures; 2020. Disponible en: https:// www.nlm.nih.gov/bsd/uniform requirements.html\#electronic

30. Abreu-Hernández LF, Cid-García ÁN, Herrera-Correa G, Manuel JV, Laviada-Delgadillo R, Rodríguez C, Sánchez-Aparicio JA. Perfil de competencias del médico general mexicano. México: Asociación Mexicana de Facultades y Escuelas de Medicina A. C.; 2008.

31. Olivares S, Rivera N, López M y Turrubiates M. Etapas de la identidad para ser profesionista: Evolución de las expectativas de los retos académicos a lo largo de la carrera. Form Univ. 2020; 13(4).

32. Gruppen LD, Irby DM, Durning SJ, Maggio LA. Conceptualizing learning environments in the health professions. Acad Med. 2019;94:969-974.

33. Esperón- Hernández R. ¿Las escuelas de medicina se deben ocupar en las competencias emocionales de sus estudiantes? Rev Inv Edu Med. 2018;7:10-12.

34. Leo-Ramírez C, Zapata-González A, Esperón-Hernández R. Estudio y desarrollo de las competencias emocionales en estudiantes de medicina: Una aproximación bibliométrica. Rev Inv Edu Med. 2019;3:92-102.

35. González-Amarante P, Olivares-Olivares S, Fouquet A. La visión de los estudiantes de medicina sobre su profesión: obstáculos para la transición a la perspectiva centrada en el paciente. Edu Med. 2019 Nov 27. DOI: 10.1016/j.edumed.2019.09.006

36. Clithero-Eridon A, Albright D, Ross A. Conceptualising social accountability as an attribute of medical education. Afr J Prim Health Care Fam Med. 2020 Feb 18:12:e1-e8. doi: 10.4102/phcfm.v12i1.221

37. Woolliscroft JO. Innovation in response to the COVID-19 pandemic crisis. Acad Med. 2020 Apr 8;10.1097/ACM.0000000000003402. DOI: 10.1097/ ACM.0000000000003402.

38. Valdez-García JE. Brevísima historia de la educación médica. Rev Avances. 2019;1:37-38

39. Olivares-Olivares SL, Valdez-García JE. Aprendizaje centrado en el paciente: cuatro perspectivas para un abordaje integral. México: Editorial Médica Panamericana; 2017.

40. Sundberg K, Reeves S, Josephson A, Nordquist J. Framing IPE. Exploring meanings of interprofessional education within an academic health professions institution. J Interprof Care. 2019;33:628-635.

41. Koehn ML and Charles SC. A Delphi study to determine leveling of the interprofessional core competencies for four levels of interprofessional practice. Med Sci Edu. 2019;29:389-398.

42. Kofman F. La empresa consciente. Argentina: Aguilar; 2016.

43. Taeli-Gómez F. El nuevo paradigma de la complejidad y la educación: una mirada histórica. Polis Rev Latinoam. 2010;25:1-13.

44. Fawns $\mathrm{T}$, Jones $\mathrm{D}$, Aitken G. Challenging assumptions about "moving online" in response to COVID-19, and some practical advice. MedEdPublish 2020 Abr 30. DOI: 10.15694/mep.2020.000083.1

45. White RH. Report on the Future of Higher Education. EE. UU: OSRE; 2018.

46. Boelen C, Dharamsi S, Gibbs T. The social accountability of medical schools and its indicators. Edu Health (Abingdon). 2012;25:180-194.

47. Torre G. COVID-19: salud y economía. Reforma (México). 2020 May 3. 